\title{
The Use of Vocabulary Learning Strategies in Relation to Vocabulary Size of Students in Chinese Universities
}

\author{
Tian Tian \\ School of Foreign Languages \& Cultures, Beijing Wuzi University, Beijing, China \\ Email address: \\ xiaotian21121@sina.com

\section{To cite this article:} \\ Tian Tian. The Use of Vocabulary Learning Strategies in Relation to Vocabulary Size of Students in Chinese Universities. Communication and \\ Linguistics Studies. Vol. 5, No. 4, 2019, pp. 80-85. doi: 10.11648/j.cls.20190504.12
}

Received: December 16, 2019; Accepted: December 31, 2019; Published: January 8, 2020

\begin{abstract}
The study tends to explore vocabulary learning beliefs and strategies employed by students in Chinese universities. The correlations between beliefs/strategies and students' vocabulary size are also addressed to show the impact of the different vocabulary learning strategies on students' vocabulary size in China. A questionnaire and a vocabulary test were administered to 105 students from two universities. The questionnaire is adapted from $\mathrm{Gu}$ and $\mathrm{Hu}[1]$ and the vocabulary levels test is selected from Nation's [2] vocabulary levels test (the university word list level) to evaluate students' vocabulary size. With SPSS, statistical descriptions and pearson correlation are undertaken to process the data collected. The analyses of the data reveal that students in Chinese universities are interested in learning vocabulary. They report adopting a number of learning strategies in their vocabulary learning processes. They appear to use metacognitive strategies more often when they learn vocabulary, especially self-initiation. At cognitive level, they are both inclined to adopt dictionary use, guessing, note-taking and activation strategies very often, while encoding and rehearsal are less used. Their vocabulary size has a strong relation with their strategy use at cognitive level, a moderate relation at overall and metacognitive level and no relation with their learning beliefs. The study suggests that teachers should encourage students to hold a positive belief on vocabulary learning and to employ both metacognitive and cognitive strategies in learning vocabulary. Students should pay as much attention to cognitive strategies as to metacognitive strategies.
\end{abstract}

Keywords: Vocabulary Learning Beliefs, Vocabulary Learning Strategies, Vocabulary Size

\section{Introduction}

Just as Wilkins [3] put it, "Without grammar very little can be conveyed; without vocabulary nothing can be conveyed". Krashen and Terrell [4] indicated that vocabulary is of prime concern in second language context for its dominant role in academic achievements. Rivers [5] illustrated that vocabulary is essential for second language learning because without an extensive vocabulary, we can't use the structure we want for comprehensible communication. According to Hermann [6], language for us would be reduced to a mere discourse of iconic gestures and symbols if there are no words. Cook [7] held that grammar provided the overall patterns while vocabulary provided the material to put in these patterns. It is the vocabulary that makes the communication feasible under certain circumstances. Vocabulary plays an important role in second language learning. Vocabulary is the very basis of learning a language. The size of one's vocabulary will have an effect on one's language proficiency.

Learning strategies are of great account while learning a language. Oxford [8] indicates that: (1) Strategies are tools for active, self-directed involvement, which is essential for developing communicative competence; (2) Appropriate learning strategies result in improved proficiency and greater self-confidence. Therefore, the knowledge of learning strategies is important because it will facilitate language learning considerably. As vocabulary is so important in second language acquisition, the strategies adopted by successful learners in vocabulary learning is worth discussing. Researchers [9-11] also believe teaching vocabulary learning strategies is the best way to teach learners vocabulary. Some linguists and educators [12-13] have already begun their studies on vocabulary learning strategies in China.

In order to be active in vocabulary learning, students should be aware of vocabulary learning strategies they use and trained in specific strategies. The purpose of the research is to 
describe different beliefs and vocabulary learning strategy patterns students may use in Chinese universities and the relationship between vocabulary learning strategies and students' vocabulary size. The influence of students' vocabulary learning strategies on vocabulary can help teacher train students to use some strategies to memorize vocabulary efficiently and quickly while learning English.

\section{Literature Review}

\subsection{Vocabulary Learning Strategies}

\subsubsection{Definition of Vocabulary Learning Strategies}

A general definition for the term strategy in linguistics can be "a mental or behavioral activity related to some specific stage in the overall process acquisition or language use" [14]. Another widely-accepted definition for language learning strategies is from Oxford [8], who claimed "learning strategies are specific actions taken by the learner to make learning easier, faster, more enjoyable, more self-directed, and more transferable to new situations".

From the above definitions, vocabulary learning strategies can be defined as approaches that students use to improve their learning while acquiring the vocabulary. Schmitt [10] explained vocabulary learning strategies following Rubin's words "the process by which information is obtained, stored, retrieved, and used", therefore vocabulary learning strategies could be quite broad. He also proposed the taxonomy of vocabulary learning strategies based on Oxford's [8] social, memory, cognitive, and metacognitive strategies. Schmitt introduced another determination strategies and excluded affective and compensation strategies. The two-dimensional taxonomy includes discovery strategies and consolidation strategies. According to Nation [15], vocabulary learning strategies are divided into three subcategories as "planning", "source" and "processes". Planning involves deciding on where, how and how often to focus attention on the vocabulary item. Source relates to getting information about the word. Process tries to build word knowledge through noticing, retrieving and generating.

\subsubsection{Strategy Use in Learning Vocabulary}

Many studies have shown use of learning strategies when learning a language [12, 16-17]. There exist different opinions about the most commonly used strategies and the effectiveness of some strategies. Ahmed [18] figured out five kinds of learners on studying 300 Sudanese learners of English, according to the strategies they used. "Good learners" used vocabulary learning strategies more. Fan's [19] study aimed to investigate the frequency of use, perceived usefulness, and actual usefulness of second language vocabulary learning strategies of 1067 learners in Hong Kong.

A lot of research has been conducted on dictionary use while leaning vocabulary. Scholfield [20] proposed seven steps for learning and analyzed each step in detail. He argued using a dictionary should not be only a technical and passive activity, it is a complex process involving the active participation of the learner. Schmitt [10] inquired the actual strategy use and perceived helpfulness of 600 Japanese students company employees. The "winner" was inclined to a bilingual dictionary and other vocabulary learning strategies took the order as: written and verbal repetition, study spelling of a word, and guessing from context. Kudo's [21] study were involved with Japanese senior high school students is in accordance with Schmitt's [10] findings.

Another view is that contextual guessing is the most favored type of strategy. Nation [9] focused on three vocabulary learning strategies: guessing in context, using mnemonic techniques and using prefixes, roots, and suffixes to learn or guess unfamiliar words. He regarded guessing from context as the "undoubtedly most important vocabulary learning strategy" [9]. Oxford and Scarcella [22] held a similar view when they refer to comprehension in reading that the most useful vocabulary learning strategy is contextual guessing. Liu Jinkai [13] studied the vocabulary learning strategy "guessing meaning". He argued that guessing meaning played an important role in students' acquisition of vocabulary.

Ahmed [18] discovered that note-taking is a strategy Sudanese learners used very frequently, but significant difference does not exist between the good learners and the poor learners. Empirical research showed that it is necessary to use repetition strategies on vocabulary learning. Schmitt [10] investigated Japanese students and found rote repetition ranked highly. Some scholars [4, 23] found that extensive reading is an effective strategy when learning vocabulary. Wang [24] concluded that Chinese EFL learners employ a variety of memorization strategies and a high relation exists between their adoption of memorization strategies and their performance in the word recall tests. Levin compared the keyword method with context method and finds that subjects in the keyword condition performed better than subjects in the contextual condition [cited in 23].

Khoii and Sharififar [29] conducted a study on the effects rote memorization and semantic mapping while learning vocabulary. They divided 38 participants into two groups. One used rote memorization and the other used semantic mapping strategy. A vocabulary test was given to measure the learning outcomes respectively after four-month treatment. No significant differences were found from the results. Semantic mapping strategy was not superior to rote memorization for learners' vocabulary acquisition.

\subsection{Relationship Between Vocabulary Learning Strategies and Learning Achievement}

Many researches have been done to show the relationship between vocabulary learning strategies and vocabulary size. $\mathrm{Gu}$ and Johnson [17] investigated the vocabulary learning strategies used by 850 non-English majors at a university in China and the relationship between strategies, vocabulary size, and language proficiency. Contextual guessing, the skills of using dictionaries, note-taking and activation of newly learned words correlated positively with the two test scores, but visual repetition of new words was the strongest negative predictor of both vocabulary size and general proficiency. Furthermore, 
strategies for retaining vocabulary just related more to vocabulary size than to general English learning.

Wang [24] employed a quantitative approach and a vocabulary test to investigate 50 Chinese junior students in Nanjing University. The results revealed that Chinese students did not believe that words could be naturally acquired. They employed a variety of strategies to facilitate word memorization including management strategy, word list strategy, repetition strategy, encoding strategy and application strategy. He also found significant correlation between strategy use and the students' performance in word recall tests.

Kojic-sabo and Lightbown [25] conducted a survey focusing on the relationship between vocabulary learning strategies use and learning outcomes. The findings of their study suggest a strong relationship between the amount of strategy use and levels of success in language learning. Also they found that time spending on vocabulary active use of the target language outside the classroom is associated with better learning outcomes.

Zhang [26] found out the difference between the stages of general academic English learning and the professional English learning. She found that the graduates in the stage of general academic English learning used more strategies and used more frequently than those in the stage of professional English learning. There was almost no difference in the use of metacognitive strategies between the two stages, while the social/affective strategies were used more frequently in the stage of general academic English learning. The strategies of translation/mother tongue were used more frequently in the professional English learning stage. The vocabulary learning strategies had strong correlation with vocabulary achievement at both stages.

Atasheneh Nasser and Naeimi Maki [30] investigated the effect of vocabulary learning strategy by adopting mechanical techniques in reading comprehension by Iranian learners. The results showed that mechanical techniques vocabulary learning strategy could lead a better vocabulary achievements by Iranian learners.

\section{Methodology}

\subsection{Participants}

The participants in the survey were 105 students from Dalian Maritime University and Tianjin Foreign Studies University in China. The age range of these participants was 19-21. They were second-year and third-year students who all have studied English for 10-12 years since Chinese students begin learning English when they are in the primary school. They were from two departments: Marine Engine Engineering (from Dalian Maritime University) and Educational Technology (from Tianjin Foreign Studies University).

\subsection{Instruments}

The study included a questionnaire for investigating the students' vocabulary learning strategies and a vocabulary levels test that assessed the participants' vocabulary knowledge or vocabulary size.

The vocabulary learning questionnaire (VLQ Version 5) [1], written in Chinese, has two sections including three dimensions, which are beliefs about vocabulary learning, metacognitive strategies and cognitive strategies. Participants have to rate each statement on a 7-point scale from Absolutely Disagree (1) to Absolutely Agree (7). Thus higher scores indicate greater use of the strategy.

Table 1 outlines the major dimensions in the questionnaire, the categories under each dimension, the number of items under each category, and the internal consistency reliabilities of each category tested by Gu successively [1].

Table 1. Dimensions and categories of VLS questionnaire by Gu (2003).

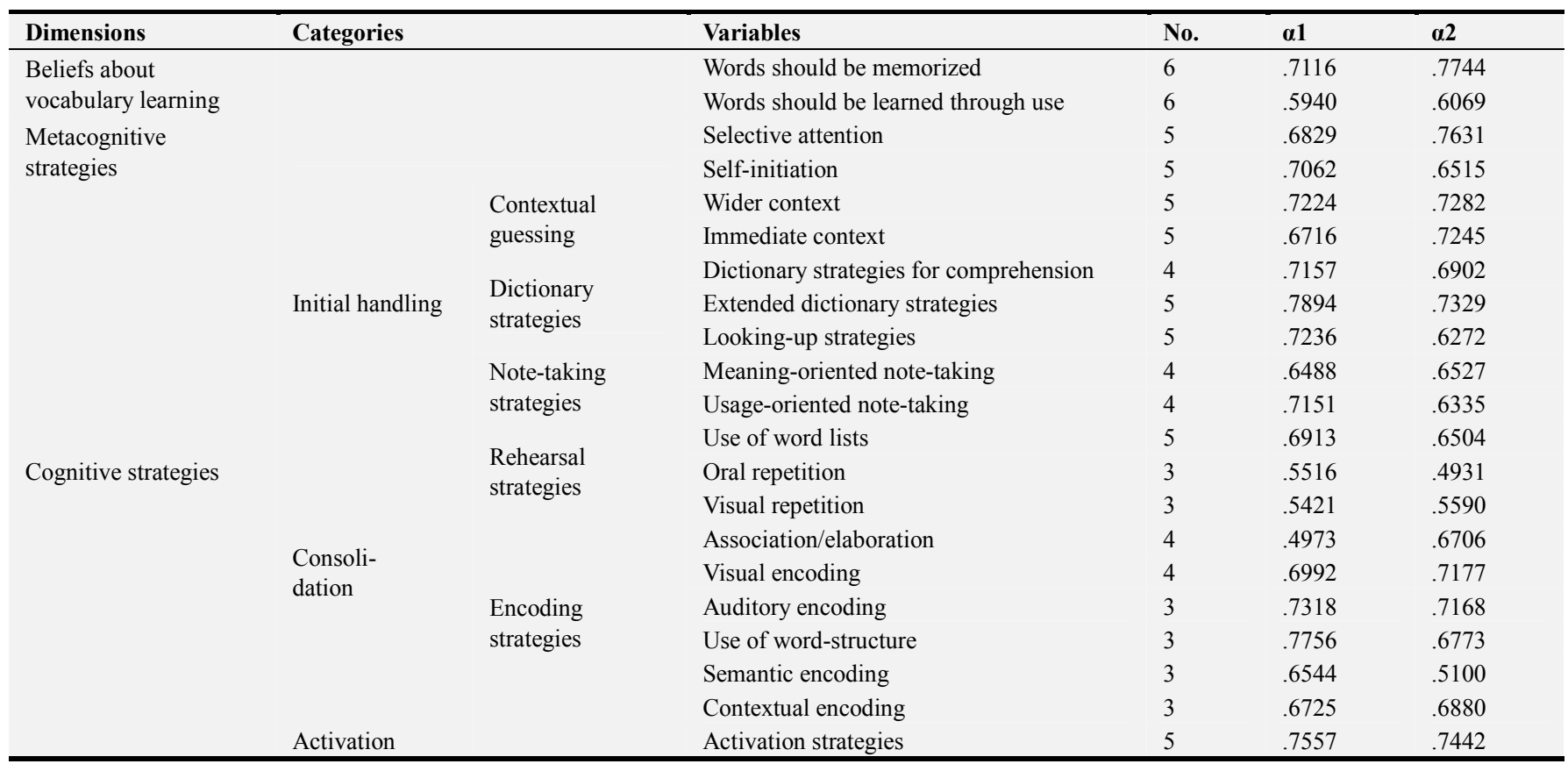


The vocabulary recall test is selected from Nation's Vocabulary Levels Test [2]. The original includes five parts: the 2,000-word level, the 3,000-word level, the 5,000-word level, the university word list level and the 10,000-word level. The test involves word-definition matching in a reversal of the standard practice. The participants are required to match the three definitions with three of the six words provided by writing the numbers of the words before the corresponding definitions, for example:

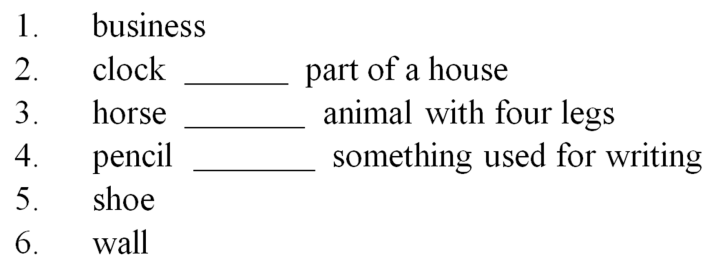

The answer is in the following way:

$\begin{array}{lll}\text { 1. } & \text { business } & \\ \text { 2. } & \text { clock } \frac{6}{6} \text { part of a house } \\ \text { 3. } & \text { horse } \frac{3}{4} \text { animal with four legs } \\ \text { 4. } & \text { pencil } \frac{4}{4} \text { soming used for writing } \\ \text { 5. } & \text { shoe } & \\ \text { 6. } & \text { wall }\end{array}$

The survey employed the Vocabulary Levels Test at the university word level as our vocabulary size indicator. Scores summed the number of correct responses. The full mark of this test is 18 points, with each item scored one point.

\subsection{Data Analyses}

The survey was undertaken in Dalian Maritime University in September, 2018 and in Tianjin Foreign Studies University in October, 2018. The participants were encouraged to finish the questionnaire. After the questionnaire came the vocabulary levels test. It took about 20 minutes in all. Both were collected within the set time.

91 students provided qualified questionnaires. The data was entered into computer and analyzed by SPSS. Descriptive statistics such as means, standard deviations were obtained first to see the overall patterns of vocabulary learning strategies adopted by Chinese university students. Then correlation analyses had been conducted between the three independent variables and the dependent variable - vocabulary size, to see how various strategies affect students' vocabulary size in Chinese context.

\section{Results and Discussions}

This study tries to figure out different beliefs and vocabulary learning strategies Chinses university students may use and the relationship between students' vocabulary learning strategies and vocabulary size. It addresses both Chinese students' vocabulary learning beliefs and vocabulary learning strategies, and correlation analyses between vocabulary learning strategies and vocabulary size respectively.

\subsection{Beliefs and Vocabulary Learning Strategies of Chinese University Students}

The internal reliability of the questionnaire is .923 . The mean value $(\mathrm{M}=4.2737, \mathrm{SD}=.5412)$ of overall vocabulary learning strategies use shows Chinese students often use vocabulary learning strategies when they meet new words, because it is higher than medium score 4 . They hold the opinion that it is necessary to make use of these strategies in their vocabulary learning.

Mean value and standard deviation of beliefs about vocabulary learning are calculated as the table listed below. Table 2 shows the beliefs of Chinese university students in their vocabulary learning.

Table 2. Mean and standard deviation of non-English majors' beliefs on vocabulary learning.

\begin{tabular}{llcc}
\hline Categories of beliefs & No. of items & Mean & SD \\
\hline Beliefs about vocabulary learning & 12 & 4.2894 & .5302 \\
Words should be memorized & 6 & 3.3736 & .9157 \\
Words should be learned through use & 6 & 5.2051 & .6912 \\
\hline
\end{tabular}

As shown in Table 2, beliefs on vocabulary learning place a mean of 4.2894, showing students in China are interested in learning vocabulary and they are inclined to use various strategies to acquire new words. "Words should be learned through use" is much more popular than "words should be memorized", similar to some previous studies [1, 12]. Chinese students prefer to learn English vocabulary by using them to memorizing them.

Table 3. Mean and standard deviation of non-English majors' metacognitive strategies.

\begin{tabular}{llll}
\hline Categories of strategies & No. of items & Mean & SD \\
\hline Metacognitive strategies & 10 & 4.3571 & .7344 \\
Selective attention & 5 & 4.1055 & .9644 \\
Self-initiation & 5 & 4.6088 & .9775 \\
\hline
\end{tabular}

Metacognitive strategies are often used by Chinese university students (Table 3). At metacognitive level, both selective attention and self-initiation are scored above 4, indicating both of the two categories ar used very often, especially self-initiation. Self-initiation has the higher value, in accordance with Gu and Johnson's study [17].

Table 4 indicates general descriptive statistics on the vocabulary strategy use at the dimension of cognitive strategies with mean and standard deviation of each category.

Table 4. Mean and standard deviation of non-English majors' cognitive strategies.

\begin{tabular}{lllll}
\hline Categories of strategies & $\begin{array}{l}\text { No. of } \\
\text { items }\end{array}$ & Mean & SD & Rank \\
\hline Cognitive strategies & 68 & 4.2587 & .6185 & \\
Contextual guessing & 10 & 4.4736 & .7915 & 3 \\
Wider context & 5 & 4.6374 & .8579 & \\
Immediate context & 5 & 4.3099 & .9263 & \\
Dictionary strategies & 14 & 4.6947 & .8293 & 1 \\
\hline
\end{tabular}




\begin{tabular}{lllll}
\hline Categories of strategies & $\begin{array}{l}\text { No. of } \\
\text { items }\end{array}$ & Mean & SD & Rank \\
\hline Dictionary strategies for & 4 & 4.6868 & 1.1009 & \\
comprehension & 5 & 4.9033 & 1.0299 & \\
Extended dictionary strategies & 5 & 4.4923 & .9993 & \\
Looking-up strategies & 8 & 4.5000 & .9188 & 2 \\
Note-taking strategies & 4 & 4.4093 & 1.0623 & \\
Meaning-oriented note-taking & 4 & 4.5907 & .9864 & \\
Usage-oriented note-taking & 4 & 3.8871 & .8731 & 6 \\
Rehearsal strategies & 11 & 3.7385 & 1.0395 & \\
Use of word lists & 5 & 4.2418 & 1.1569 & \\
Oral repetition & 3 & 3.7802 & 1.2435 & \\
Visual repetition & 3 & 3.9473 & .8827 & 5 \\
Encoding strategies & 20 & 4.1099 & 1.0309 & \\
Association/ elaboration & 4 & 3.7473 & 1.15920 & \\
Visual encoding & 4 & 3.9231 & 1.4308 & \\
Auditory encoding & 3 & 3.9084 & 1.2263 & \\
Use of word-structure & 3 & 3.7692 & 1.1618 & \\
Semantic encoding & 3 & 4.2381 & 1.0126 & \\
Contextual encoding & 3 & 4.2587 & .6185 & 4 \\
Activation strategies & 68 & & & \\
\hline
\end{tabular}

It is obvious that dictionary use strategy ranks the highest not only among the cognitive strategies but also among all the vocabulary learning strategies. Chinese students tend to consult dictionaries when the unfamiliar words appear. Making use of dictionaries is also much valued by researchers. Allen [27], for instance, said that dictionaries were passports to independence. They adopt extended dictionary strategies, dictionary strategies for comprehension and looking-up strategies. Extended dictionary strategies rank the highest among all the strategies. The extended strategies demonstrate examples in dictionaries are of course essential both to extend the user's comprehension and to provide models for students to remember, perhaps eventually produce by putting individual words into a range of typical contexts and appropriate phrases.

Note-taking ranks as the second most frequent strategies. Also notable is that usage-oriented note-taking is used at a high level, which is higher than the score 4.5. Students attach greater importance to the usage of the words than the meaning of the words.

The strategies next to note-taking are contextual guessing strategies, which are also often used. Both wider context and immediate context are above the medium level 4, especially wider context scored larger than 4.5 , with a higher frequency in use.

Activation strategies rank the fourth among cognitive strategies, but higher than medium level 4. Ghazal [28] explained that activation strategies include those strategies through which the learners actually use new words in different contexts. For instance, learners may set sentences using the words they have just learned. The strategies are in line with their belief that words should be learned through use and context. It shows that the students realize that they should use the strategy in vocabulary learning.

Among encoding strategies, visual encoding, auditory encoding, use of word-structure and semantic encoding are less popular, but the association and contextual encoding are used often. According to the data, Chinese students don't put the language in use very often even though they know they should, according to their beliefs in vocabulary learning. However, they are conscious of contextual information and words of related meaning.

In the case of rehearsal strategies, as shown in the above table, students seem to use more oral repetition than word lists and visual repetition. Rehearsal strategies are also the least used by non-English majors in the survey, as Nation [2] mentioned, "Some psychologists believe that repetition is not an important factor in vocabulary learning."

\subsection{The Relationship Between Vocabulary Learning Strategies and Vocabulary Size}

The author performed the correlation analysis between two variables, vocabulary learning strategies and vocabulary size to find out how vocabulary learning strategies affect vocabulary learning strategies. Simple correlations were obtained between four dependent variables (overall patterns and three dimensions as beliefs, metacognitive strategies and cognitive strategies) and one dependent variable (vocabulary size according to the students' scores in the vocabulary levels test).

Table 5. Correlations between vocabulary learning strategies and vocabulary size of Chinese students.

\begin{tabular}{|c|c|c|c|c|}
\hline & Overall patterns & Beliefs & Metacognitive strategies & Cognitive strategies \\
\hline Pearson Correlation & $.346(* *)$ & .115 & $.216(*)$ & $.346(* *)$ \\
\hline Sig. (2-tailed) & .001 & .279 & .040 & .001 \\
\hline
\end{tabular}

* Correlation is significant at the 0.05 level (2-tailed).

** Correlation is significant at the 0.01 level (2-tailed).

In table 5, a moderate positive relationship $(\mathrm{r}=.346, \mathrm{p}=.001)$ is found between the overall patterns of vocabulary learning strategies and vocabulary size, showing that Chinese students enlarge their vocabulary size by employing vocabulary strategies. Knowing and using some strategies can better facilitate students to acquire English words.

The correlation analysis indicates that vocabulary size is not statistically correlated with the beliefs they hold $(r=.115$, $\mathrm{p}=.279$ ), which implies that the learning beliefs have no significant influence on their vocabulary learning. The relationship between metacognitive strategies and vocabulary size of the students is moderately positive $(\mathrm{r}=.216, \mathrm{p}=.040)$. That means if the students are conscious of using metacognitive strategies, they will do well in vocabulary learning to some extent. A strong positive correlation is found between cognitive strategies and vocabulary size of them, showing cognitive strategies have the most influence on vocabulary learning.

\section{Conclusion}

As far as the overall pattern of vocabulary learning strategies is concerned, students from Chinese universities use 
vocabulary learning strategies very often. Among the two levels of vocabulary learning strategies, the use of metacognitive strategies is the highest, and cognitive strategies follows. The result of rank order is in accordance with what Ellis [14] have found in their studies.

According to the descriptive statistics of learners' vocabulary learning strategies, teachers should know the students' current beliefs and strategies and provide more systematic instruction on vocabulary learning for the students while teaching English vocabulary. This can be done through surveys, interviews, etc.

To the different stress on vocabulary learning strategies, teachers should pay much more attention to encoding and rehearsal strategies because the students use encoding and rehearsal strategies less often. They are not good at consolidating their vocabulary learning outcomes. Teachers are advised to help the students in this area.

In light of results found in the survey, the use of metacognitive strategies ranks first and cognitive strategies second. Thus students are firstly conscious of controlling vocabulary learning as a general process. However, from the correlation result between vocabulary learning strategies and vocabulary size, cognitive strategies have greater influence on students' vocabulary proficiency. Students are expected to improve their cognitive strategies in vocabulary learning.

\section{References}

[1] Gu, Yongqi, Hu, Guangwei. (2003). Empirical Study on English Learning Strategies. Shaan'xi Normal University Press.

[2] Nation, I. S. P. (2004). Teaching and learning vocabulary. Beijing: Foreige Language Teaching and Research Press.

[3] Wilkins, D. A. (1972). Linguistics in Language Teaching. London: Edward Arnold.

[4] Krashen, S. and Terrell, T. (1983). The natural approach: Language acquisition in the classroom. Oxford: Pergamon.

[5] Rivers, W. (1983). Connnunicating Naturally in a Second Language. Cambridge: Cambridge University Press.

[6] Hermann, F. (2003). Differential Effects of Reading and Memorization of Paired Associates on Vocabulary Acquisition in Adult Learners of English as a Second Language. Teaching English as a Second or Foreign Language, 7: A1.

[7] Cook, V. (2000). Linguistics and Second Language Acquisition Beijing: Foreign Language Teaching and Research Press.

[8] Oxford, R. (1990). Language Learning Strategies: What Every Teacher Should Know. New York: Newbury House.

[9] Nation, P. (1990). Teaching and learning vocabulary. Boston: Heinle and Heinle.

[10] Schmitt, N. (1997). Vocabulary learning strategies. In N. Schmitt and M. McCarthy (eds.) Vocabulary: Description, Acquisition, and Strategies. Cambridge: Cambridge University Press.

[11] Lawson, M. and Hogben D. (1996). The vocabulary-learning strategies of foreign-language students. Language Learning, 46: 101-135.
[12] Wu, Xia, and Wang, Qiang. (1998). The Vocabulary Learning Strategies of Non-English Majors. Foreign Language Teaching and Research (1), 53-57.

[13] Liu, Jinkai. (1999). Research on Language Learning strategies-Word Guessing and Language Proficiency. Foreign Language Education (3), 31-35.

[14] Ellis, R. (1994). The Study of Second Language Acquisition. Oxford: Oxford University Press.

[15] Nation, P. (2001). Learning vocabulary in another language. Cambridge: Cambridge University Press

[16] Carter, R. and M. McCarthy. (1988). Vocabulary and Language Teaching. New York: Longman Press.

[17] Gu, Yongqi and R. K. Johnson. (1996). Vocabulary learning strategies and language learning outcomes. Language Learning, 46: 643-679.

[18] Ahmed, M. O. (1989). Vocabulary learning techniques. In P Meara (Ed.), Beyond Words. London: CIL: 3-14.

[19] Fan. (2003). Frequency of use, perceived usefulness, and actual usefulness of second language vocabulary strategies: A study of Hong Kong learners. The Modern Language Journal, 87 (2): 222-241.

[20] Scholfield, P. (1982). Using the dictionary for comprehension. TESOL Quarterly, 16 (2): 185-194.

[21] Kudo, Y. (1999). L2 vocabulary learning strategies (nflrc network 14) [html document]. [online]. Available: http://www.lll.hawaii.edu/nflrc/NetWorks/NW14/.

[22] Oxford, R. and R. Scarcella. (1994). Second Language Vocabulary Learning Among Adults: State of the Act in Vocabulary Instruction. System, 22: 231-243.

[23] Coady, J and T. Huckin (eds.). (1997). Second Language Vocabulary Acquisition. Cambridge: Cambridge University Press.

[24] Wang, Wenyu. (1998). Beliefs, Strategies and English Vocabulary Retention [J]. Foreign Language Teaching and Research (1). 49-54.

[25] Kojic-Sabo, I. and P. M. Lightbown. (1999). Students' approaches to vocabulary learning and their relationship to success. Modern Language Journal, 83: 176-192.

[26] Zhang, Ping. (2001). A Research on Learning Strategies of Postgraduates' Basic and Professional Vocabulary. Foreign Language Teaching and Research. (6), 442-449.

[27] Allen, V. F. (1983). Techniques in teaching vocabulary. Oxford: Oxford University Press.

[28] Ghazal, L. (2007). Learning Vocabulary in EFL Contexts Through Vocabulary Learning Strategies. [online]. Available: $\mathrm{http}: / /$ novitasroyal.org/Ghazal.pdf

[29] Khoii, R. \& Sharififar, S. (2013). Memorization versus semantic mapping in L2 vocabulary acquisition. ELT journal, 67 (2), 199-209.

[30] Atasheneh Nasser \& Naeimi Maki. Vocabulary Learning through Using Mechanical Techniques Vocabulary Learning Strategy [J]. Theory and Practice in Language Studies, 2015, 5 (3): 541-548. 